\title{
Simultaneous Use of Both Bilateral Intralaminar and Pedicle Screws for C2 Stabilization
}

\author{
Kiyoshi Tarukado, Osamu Tono, Toshio Doi \\ Department of Orthopedic Surgery, Kyushu University Beppu Hospital, Oita, Japan
}

Four patients underwent stabilization surgery using both bilateral C2 pedicle screw (PS) and intralaminar screw (LS). Neural and vascular injury resulting from incorrect screw placement was assessed using computed tomography (CT). The evaluation of bone union was assessed by lateral flexion-extension X-ray films and CT. The symptoms were improved in all patients. There were no intraoperative complications. Furthermore, there were no cases of neurological worsening or vascular injury from incorrect screw placement. Failure of instrumentation or screw loosening during the follow-up period did not occur in any of the patients. All cases had accomplished bone union at the final follow-up. Theoretically, the stabilization technique using both bilateral C2 PS and LS at the same time can provide more stability than any other single technique. Simultaneous use of both bilateral C2 PS and LS is potentially a good choice for surgical repair.

Keywords: Spinal fusion; Pedicle screws; Laminar

\section{Introduction}

Upper cervical lesions are often closely related to instability. These pathological conditions are caused by various conditions, including trauma, congenital anomalies, neoplasms, infections, and inflammatory diseases. As a result, patients suffer from paralysis and pain due to instability. Such clinical conditions require a stabilization technique involving the $\mathrm{C} 2$ vertebra in order to improve the patients' symptoms. An ideal stabilization method can simultaneously provide both safe and rigid fixation.

However, the use of stabilization techniques in the upper cervical spine involving the $\mathrm{C} 2$ vertebra is associated with some anatomical difficulties. For example, the vertebral artery (VA) and spinal cord are located near the trajectory of the screw. In addition, there is a wide range of motion in the upper cervical spine. Panjabi et al. [1] re- ported that the greatest degree of flexion occurs at $\mathrm{C} 1-\mathrm{C} 2$ and the greatest degree of extension is observed at $\mathrm{C} 0-\mathrm{C} 1$, and the greatest degree of rotation is recorded at $\mathrm{C} 1-\mathrm{C} 2$. These problems make the safety and rigid fixation involving C2 vertebra difficult. Various stabilization methods that address these problems have been reported [2-5].

Currently, intralaminar screw (LS) has been widely accepted due to their safety. Many biomechanical and safety evaluations comparing pedicle screw (PS) and LS have been performed [6-10]. The biomechanical characteristics of LS resembles that of PS, however, PS is superior to LS in some biomechanical points. In contrast, LS is considered safer than PS with regard to VA injury. The most suitable stabilization technique is unclear. Theoretically, a stabilization method using both bilateral C2 PS and LS at the same time would provide more stability than the single technique. The shape of the C2 facilitates simulta-

Received Dec 10, 2014; Revised Jan 8, 2015; Accepted Feb 2, 2015

Corresponding author: Toshio Doi

Department of Orthopedic Surgery, Kyushu University Beppu Hospital,

4546 Tsurumihara, Tsurumi Beppu, Oita 874-0838, Japan

Tel: +81-977-27-1755, Fax: +81-977-27-1755, E-mail: toshidoi@ortho.med.kyushu-u.ac.jp 
Table 1. Summary of clinical findings in four patients treated both bilateral PS and LS

\begin{tabular}{lcccccc} 
Patient & Age (yr)/sex & $\begin{array}{c}\text { Clinical } \\
\text { symptom }\end{array}$ & $\begin{array}{c}\text { Motor } \\
\text { palsy }\end{array}$ & $\begin{array}{c}\text { Urinary } \\
\text { disturbance }\end{array}$ & $\begin{array}{c}\text { Radiological } \\
\text { diagnosis }\end{array}$ & Complication \\
\hline 1 & $58 /$ female & Myelopathy & + & - & AAS+VS & RA \\
2 & $73 /$ female & Myelopathy & + & - & Cervical kyphosis & Severe osteoporosis \\
3 & $75 /$ male & Myelopathy & + & - & AAS+pseudotumor & Cerebral palsy \\
\hline 4 & $63 /$ male & Myelopathy & + & - & AAS & None \\
\hline
\end{tabular}

PS, pedicle screw; LS, intralaminar screw; AAS, atlantoaxial subluxation; VS, vertical subluxation; RA, rheumatoid arthritis.

neously insertion of both bilateral C2 PS and LS. We reported four patients that were simultaneously treated with both bilateral C2 PS and LS.

\section{Technical Note}

Between April 2011 and March 2014, four patients underwent stabilization surgery with both bilateral C2 PS and LS inserted simultaneously. The patients' age, sex, clinical symptoms, diagnosis, and preoperative complications were listed in Table 1. Prior to surgery, all patients underwent evaluation of cervical spine stability by roentgen kymograph. In addition, all patients underwent enhanced computed tomography (CT) scans to assess VA and thickness of the $\mathrm{C} 2$ lamina. The need for stabilization involving the $\mathrm{C} 2$ vertebrae was determined based on the results of these studies. Additionally, safe placement of the bilateral C2 PS and LS at the same time was feasible.

The surgical details, such as the need for decompression and the levels of stabilization, were patient-dependent. The common procedure was as follows: the patient was placed in the prone position under general anesthesia, and cervical alignment was maintained neutral to slightly extended using a Mayfield skull clamps and headrest systems (Mayfield Integra Lifescience Co., Plainsboro Township, NJ, USA). Exposure of the posterior neck from the occipital bone to the cervical vertebrae was performed in the usual manner as needed. After the venous plexus between C1-2 was carefully exfoliated, the medial margin of the $\mathrm{C} 2$ pedicle was identified. A small cortical window was opened at the insertion point using a high-speed drill. It is desirable to create an insertion hole as caudal and outward as possible, because this trajectory does not interfere with the apex of the LS. Bilateral C2 PSs were inserted along the course made as usual by probing from the insertion point while confirming the medial margin

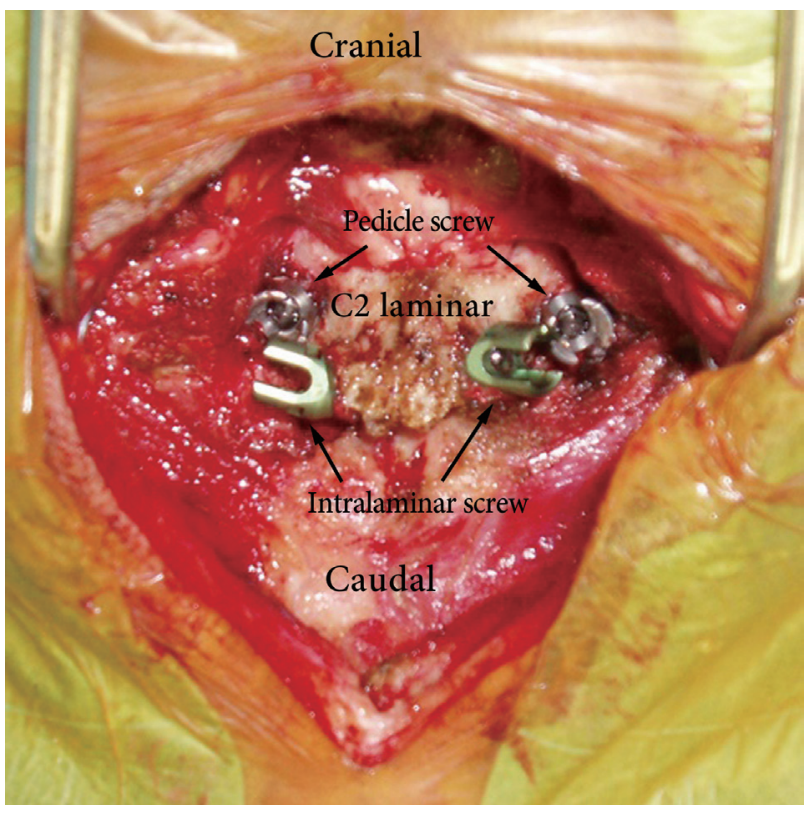

Fig. 1. A photograph of the C2 lamina after insertion of all screws.

of the C2 pedicle. The C2 LS was inserted using the technique described by Wright (Fig. 1) [5]. A small cortical window was opened at the junction of the $\mathrm{C} 2$ spinous process and lamina on the right or left, close to the cranial or caudal aspect of the C2 lamina. The contralateral lamina was carefully drilled parallel to the laminar surface using a hand drill so as not to penetrate the dorsal or ventral cortex of the lamina. After a ball probe validated the trajectory and confirmed the degree of interference with the PS, a polyaxial screw was inserted along the same trajectory. An opposite screw was also inserted, as mentioned above. If the first screw was inserted close to the cranial aspect, an opposite screw was inserted close to the caudal aspect.

After all screws were placed, the rods were bent and connected to the screws. Since it is difficult to directly 
Table 2. The size of the hardware

\begin{tabular}{|c|c|c|c|c|}
\hline Patient & Pedicle screw & Intralaminar screw & $\operatorname{Rod}(\mathrm{mm})$ & Rod material \\
\hline 1 & $\begin{array}{l}3.5 \times 28 \\
3.5 \times 28\end{array}$ & $\begin{array}{l}3.5 \times 26 \\
3.5 \times 26\end{array}$ & 3.5 & Titanium \\
\hline 2 & $\begin{array}{l}3.5 \times 28 \\
3.5 \times 28\end{array}$ & $\begin{array}{l}3.5 \times 28 \\
3.5 \times 28\end{array}$ & 3.5 & Cobalt chrome \\
\hline 3 & $\begin{array}{l}3.5 \times 24 \\
3.5 \times 32\end{array}$ & $\begin{array}{l}3.5 \times 28 \\
3.5 \times 28\end{array}$ & 3.5 & Cobalt chrome \\
\hline 4 & $\begin{array}{l}3.5 \times 32 \\
3.5 \times 32\end{array}$ & $\begin{array}{l}3.5 \times 22 \\
3.5 \times 24\end{array}$ & 3.5 & Titanium \\
\hline
\end{tabular}

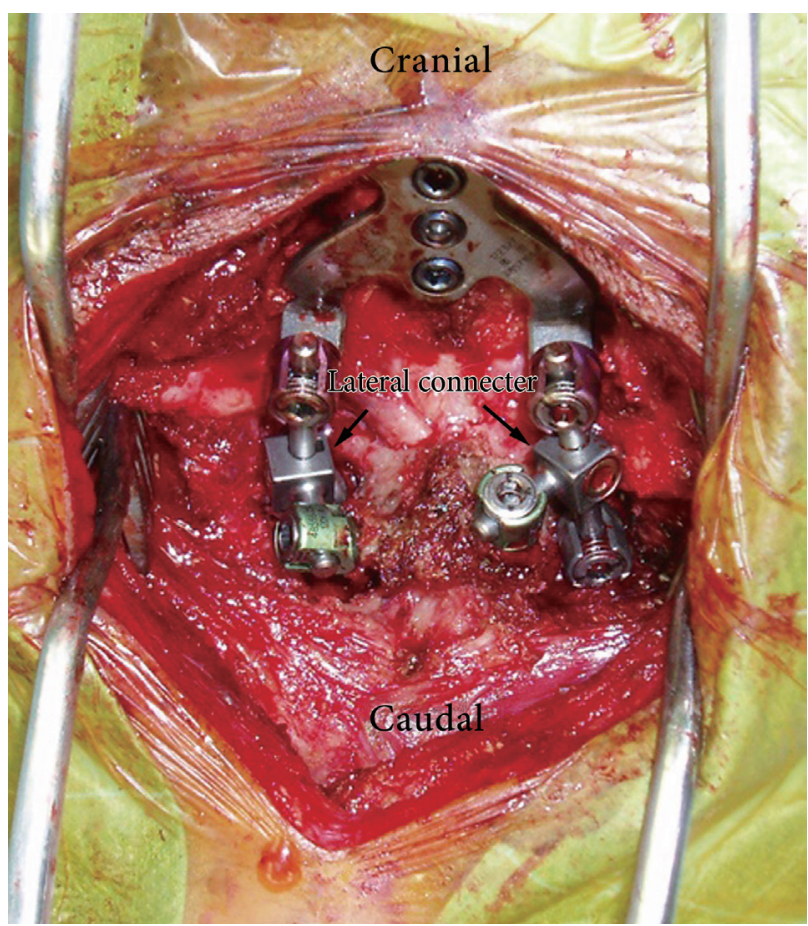

Fig. 2. A photograph of all implants after connection with the conventional lateral connectors (arrow, lateral connecter).

connect the rod to the screw, especially for C2 PS and LS, conventional lateral connecters were used to make the connection easier (Fig. 2). The details of hardware used in each patient were listed in Table 2. Finally, all laminar surfaces were decorticated for the bone graft. The bone graft was harvested from the posterior iliac crest, and adequate bone grafting was accomplished.

None of the patients were immobilized with a rigid collar after surgery. CT was performed in the immediate postoperative period to assess the accurate placement of all implants. The accuracy of the insertion of the PS was assessed using the classification proposed by Neo et al. [11]. The position of the LS was assessed in terms of whether the screws were inserted into the cortical bone. Lateral X-ray films at presurgery and postsurgery, and final follow-up were used to determine the range of motion in the fixed range.

The bone union was assessed using lateral flexion-extension X-ray films and CT, as described below. First, we determined whether the angle change between the fixed vertebral body intervals was $\leq 2$ degrees in lateral flexionextension X-ray films. Second, the presence of bridged bone was confirmed between the fixed vertebral body intervals by CT. The completion of bone union was based on whether all of the above-mentioned conditions were satisfied. The results of the imaging findings were estimated by three orthopedic surgeons and only a consensus view was taken to indicate successful bone union.

\section{Discussion}

The symptoms of all patients were improved. There were no intra- or postoperative complications, such as massive hemorrhage requiring blood transfusion, or infection. There were no cases of neurological worsening or vascular injury, including injury of the VA due to incorrect screw placement. According to the Neo's classification [11], all PS were grade 0 . With regard to the LS, penetration of the ventral laminar cortex was found for one screw. Fortunately, the screw did not worsen the patient's symptoms. All cases had accomplished bone union during the follow-up in the outpatient clinic. The mean follow-up period was 16 months (range, 12-22 months). There was no evidence of hardware failure or screw loosening on the imaging findings during the follow-up period in any of the patients. The postoperative results were summarized in Table 3. The instability before surgery was reduced after 
Table 3. Patients' characteristic after surgery

\begin{tabular}{lllcc} 
Patient & \multicolumn{1}{c}{ Operation } & Symptom & $\begin{array}{c}\text { Incorrect screw } \\
\text { placement }\end{array}$ & $\begin{array}{c}\text { Bone union } \\
\text { (mo) }\end{array}$ \\
\hline 1 & O-C2 posterior fusion & Recovery & None & + \\
\hline 2 & C2-T1 posterior fusion and C3-6 anterior fusion & Recovery & None & + \\
\hline 3 & O-C2 posterior fusion & Recovery & One LS & + \\
\hline 4 & O-C2 posterior fusion & Recovery & None & +16 \\
\hline
\end{tabular}

LS, intralaminar screw.

Table 4. Image findings in four patients treated both bilateral PS and LS

\begin{tabular}{lcccc} 
Patient & Preoperative ROM $\left({ }^{\circ}\right)$ & Postoperative ROM $\left(^{\circ}\right)$ & ROM at final follow-up $\left({ }^{\circ}\right)$ & Fixed range \\
\hline 1 & 10 & 2 & 2 & $0-C 2$ \\
2 & 53 & 2 & 1 & $\mathrm{C} 2-\mathrm{T} 1$ \\
3 & 38 & 1 & 2 & $0-\mathrm{C} 2$ \\
4 & 28 & 2 & $0-C 2$ \\
\hline
\end{tabular}

PS, pedicle screw; LS, intralaminar screw; ROM, range of motion.

surgery and maintained until the final follow-up Table 4.

Screw and rod stabilization techniques of the posterior upper cervical spine can increase the spinal stability and induce a high fusion rate. Several stabilization techniques have been developed in this field. Jeanneret and Magerl [12] reported transarticular C1/C2 fixation techniques. Their technique provides rigid fixation through the atlantoaxial joint, theoretically using tricortical screws. However, Madawi et al. [13] reported that in $20 \%$ of the cases, the VA reduces the width of the $\mathrm{C} 2$-pedicle, and these anomalies disrupt safe trans articular screw placement. In addition, this procedure was unusable for the fixed subluxation of the $\mathrm{C} 1 / \mathrm{C} 2$.

Posterior C1-C2 fusion with polyaxial screw and rod fixation was developed to solve such problems [2]. With the increasingly widespread use of this technique, C2 PS has also become popular in subaxial cervical constructs. However, it is reported that $4.1 \%$ of cases experiences crew perforation from the pedicle caused by a C2 PS [14].

The use of C2 LS is reportedly safer than PS [5]. The biomechanical characteristic of LS resemble those of PS [6-10]. However, the literature indicates that C2 LS has the risk of inducing spinal cord injury due to screws breaking out ventrally into the spinal canal [5]. The reported series had one ventral screw breakout into the spinal canal that did not lead to spinal cord injury.

The current methods are technically demanding and it is unclear that stabilization technique is the most suitable for C2 stabilization. Cases with indications for upper cervical spinal stabilization surgery with instrumentation frequently have complications such as rheumatoid arthritis, hemodialysis, cerebral palsy, or severe osteoporosis. Stabilization techniques with C2 PS or LS are familiar to spinal surgeons. Therefore, the stabilization technique of simultaneous both bilateral C2 PS and LS was applied to patients at increased risk of fractures due to such complications. This technique provided stabilization that was more rigid.

Three disadvantages associated with this technique were recognized when planning the operations. First, the rod connection is difficult. This problem was solved easily using conventional lateral connectors. Second, the bone graft bed was smaller due to the use of several implants. This problem was also solved easily by packing a bone into the gap between the laminar surface and implants. All exposed lamina surfaces are required to be well decorticated using the high-speed drill. Consequently, all patients were able to obtain bone union. Lastly, there was a risk of incorrect screw placement. This risk can be avoided by implementing adequate preoperative planning and intraoperative care. It is especially important to confirm that the width of the pedicle and lamina is adequate to insert the screws on preoperative CT. There were no post-procedural adverse events in the present series.

The adaptation of this technique involves the follow- 
ing important criteria. First, it is essential to confirm that the width of the pedicle and lamina is adequate to insert the screws on preoperative CT. An inadequate width for the screw trajectory is a contraindication. Second, patients with severe osteoporosis caused by various conditions have a higher risk of implant failure and are thus thought to be good candidates. If the patient has low risk of implant failure, it is not necessary to use the above technique; furthermore, the routine use of this technique is not recommended in all cases involving C2 stabilization. This technique is indicated for patients that fulfill both criteria. This new method is technically demanding, similar to past procedures, and is only suitable for limited cases, as described above. However, stabilization surgery simultaneously using both bilateral C2 PS and LS has an advantage over the previously reported technique using $\mathrm{C} 2$ anchors due to the rigidity and familiarity with screw insertion. Bilateral C2 PS and LS at the same time might be a good treatment choice, especially for patients with an increased risk of implant failure.

Although there may be limited cases that are suitable for simultaneous use of both bilateral C2 PS and LS, this stabilization technique theoretically provides more stability than any single technique. When more rigid stabilization is needed due to an increased risk of fracture, using both bilateral C2 PS and LS at the same time might be a good choice for treatment.

\section{Conflict of Interest}

No potential conflict of interest relevant to this article was reported.

\section{References}

1. Panjabi MM, Crisco JJ, Vasavada A, et al. Mechanical properties of the human cervical spine as shown by three-dimensional load-displacement curves. Spine (Phila Pa 1976) 2001;26:2692-700.

2. Harms J, Melcher RP. Posterior C1-C2 fusion with polyaxial screw and rod fixation. Spine (Phila $\mathrm{Pa}$ 1976) 2001;26:2467-71.

3. Nagata K, Baba S, Chikuda H, Takeshita K. Use of C2 spinous process screw for posterior cervical fixation as substitute for laminar screw in a patient with thin laminae. BMJ Case Rep 2013;2013. pii: bcr2013009889.

4. Resnick DK, Benzel EC. C1-C2 pedicle screw fixation with rigid cantilever beam construct: case report and technical note. Neurosurgery 2002;50:426-8.

5. Wright NM. Posterior C2 fixation using bilateral, crossing C2 laminar screws: case series and technical note. J Spinal Disord Tech 2004;17:158-62.

6. Benke MT, O'Brien JR, Turner AW, Yu WD. Biomechanical comparison of transpedicular versus intralaminar C2 fixation in C2-C6 subaxial constructs. Spine (Phila Pa 1976) 2011;36:E33-7.

7. Finn MA, Fassett DR, Mccall TD, Clark R, Dailey AT, Brodke DS. The cervical end of an occipitocervical fusion: a biomechanical evaluation of 3 constructs. Laboratory investigation. J Neurosurg Spine 2008;9: 296-300.

8. Nassos JT, Ghanayem AJ, Sasso RC, et al. Biomechanical evaluation of segmental occipitoatlantoaxial stabilization techniques. Spine (Phila Pa 1976) 2009; 34:2740-4.

9. Richter M, Schmidt R, Claes L, Puhl W, Wilke HJ. Posterior atlantoaxial fixation: biomechanical in vitro comparison of six different techniques. Spine (Phila Pa 1976) 2002;27:1724-32.

10. Sim HB, Lee JW, Park JT, Mindea SA, Lim J, Park J. Biomechanical evaluations of various $\mathrm{c} 1-\mathrm{c} 2$ posterior fixation techniques. Spine (Phila Pa 1976) 2011;36: E401-7.

11. Neo M, Sakamoto T, Fujibayashi S, Nakamura T. The clinical risk of vertebral artery injury from cervical pedicle screws inserted in degenerative vertebrae. Spine (Phila Pa 1976) 2005;30:2800-5.

12. Jeanneret B, Magerl F. Primary posterior fusion $\mathrm{C} 1 / 2$ in odontoid fractures: indications, technique, and results of transarticular screw fixation. J Spinal Disord 1992;5:464-75.

13. Madawi AA, Casey AT, Solanki GA, Tuite G, Veres R, Crockard HA. Radiological and anatomical evaluation of the atlantoaxial transarticular screw fixation technique. J Neurosurg 1997;86:961-8.

14. Abumi K, Shono Y, Ito M, Taneichi H, Kotani Y, Kaneda K. Complications of pedicle screw fixation in reconstructive surgery of the cervical spine. Spine (Phila Pa 1976) 2000;25:962-9. 